\title{
Maternal behaviour in American mink females with different behavioural profiles
}

\author{
D. Zieliński , B. Ślaska and I. Rozempolska-Rucińska \\ University of Life Sciences in Lublin, Institute of Biological Bases of Animal Production, \\ Akademicka 13, 20-950, Lublin, Poland
}

KEY WORDS: behaviour patterns, dams, fertility, Neovison vison, stick test

Received: $\quad 15$ June 2018

Revised: $\quad 25$ August 2019

Accepted: 20 November 2019

${ }^{1}$ Corresponding author:

e-mail: damian.zielinski@up.lublin.pl

\begin{abstract}
The aim of the experiment was to determine how the behaviour of American mink (Neovison vison) females affects their breeding results and maternal behaviour during the perinatal period. A stick test was used to evaluate the behavioural profile of 576 minks. One hundred females with a determined behavioural profile (aggressive, curious, fearful and neutral) were selected and nest building before parturition, maternal behaviour after delivery and reproduction results were examined. The preparation of the nest was assessed before the expected parturition, taking into account the necessity of human intervention in its proper construction. Maternal behaviour was observed during the first week of life of offspring. Significant differences in the fertility rate between the aggressive and neutral females with a significantly higher number of kits present at day 4 in the second type were noted. Medium quality nests were mostly observed $(60 \%)$, followed by those of very good $(37 \%)$ and poor quality $(3 \%)$. The very good quality nests were most often built by females rated as fearful. Regardless of the behaviour profile, the females spent a similar period of time with the young at the time prior to feeding (from 16.8 to $22.3 \mathrm{~min})$. So, significant differences in the number of born and raised pups in differently behaving minks can suggest that knowledge of dam behaviour can be taken into account when predicating reproduction results.
\end{abstract}

\section{Introduction}

Traits related to the hair cover and reproduction are important functional features in American mink (Neovison vison) breeding. Breeding results largely determine the profitability of mink farms (FelskaBłaszczyk et al., 2016). The results of reproduction depend not only on genetic factors (although they are features with low heritability) but also on environmental factors, i.e. the age of females, mating period, number of mattings, length of pregnancy, colour variation, nutrition or animal maintenance conditions and cage design (Malmkvist et al.,
2007; Ślaska et al., 2009; Ślaska and RozempolskaRucińska, 2011; Dawson et al., 2013; Axelsson et al., 2017; Díez-León et al., 2017). The care of offspring after the birth and the lactation period play an important role in the life of young mink as these factors affect offspring growth and development (Newberry and Swanson, 2008). The survival of young animals directly depends on the involvement of the dams in care as well as environmental conditions (temperature, design) prevailing in the nest (Tauson et al., 2006; Malmkvist et al., 2007; Zschille et al., 2010). The time the female spends for the care of the young is important not only from 
the perspective of the offspring, but also for the female herself (Meagher et al., 2012). The prolonged stay time of young with its mother in breeding conditions (in comparison to wild mink) is less favourable for the female than for the young (Dawson et al., 2013). Rearing the offspring is physiologically very burdensome for the female, especially when the litter size is big (Malmkvist et al., 2016). Young mink before weaning, as a whole, have substantially higher body weight than their mothers, therefore their demand for milk is high. Due to the high nutrition needs of pups, rearing is highly damaging for the female body and can led to female-offspring conflict (Dawson et al., 2013). The inability to move away from the offspring, as well as the lack of adequate rest, increase the occurrence of abnormal behaviour in female (stereotypies, fur chewing) (Mason and Latham, 2004) resulting in the deterioration of the female's condition and the increased susceptibility to diseases (Newberry and Swanson, 2008; Mandalaywala et al., 2014; Malmkvist et al., 2016). It was shown that the condition and weight of females after the birth of large litters decreased in line with the growth of the offspring. As the size of the litter increased, females showed less and less motivation to care for their offspring, and the occurrence of stereotypical behaviours increased (Malmkvist et al., 2016). It was also noted that dams during lactation tried to isolate themselves from kits provided they had access to the environmental enrichments, e.g., in the form of shelves onto which offspring could not climb (Dawson et al., 2013). However, such isolation is effective only for some period of time as young gets older and begins to climb up to the shelf, as it was shown by Dawson et al. (2013).

Therefore, the aim of the present study was to determine the impact of female mink temperament on breeding results and maternal behaviour during the perinatal period.

\section{Material and methods}

In the study 576 American mink (Neovison vison) females of a pastel colour variety reared on the Carnivorous Fur Animal Farm of the National Research Institute of Animal Production in Chorzelów (Poland) were used. The animals did not show any behavioural disorders. The animals were housed individually in standard mink cages $(90 \times 45 \times 45 \mathrm{~cm} ; \mathrm{L} \times \mathrm{W} \times \mathrm{H})$ with no additional environmental enrichments (shelves, straw). From the side of the corridor, the cages had a wooden folding lodge constituting a nest $(30 \times 30 \times 24 \mathrm{~cm}$; $\mathrm{L} \times \mathrm{W} \times \mathrm{H})$. The tested herd was fed in accordance with the standards for carnivorous fur animals in Poland (Gugołek, 2011). The animals were fed with wet feed based on animal-derived ingredients (cod, poultry), carbohydrates (extruded cereal grains), mineral-vitamin preparations and preservatives. Feed was placed on the top of the cage once a day (in the morning). Water was provided from an automatic water nipple system. On the farm maintaining and feeding conditions were constant during the research. Permission for the observations was obtained from the II Local Commission for Experiments on Animals in Lublin (Poland; No. 75/2014).

In order to determine the behavioural profile of the mink, the stick test was used according to the procedure described by Malmkvist and Hansen (2002). Briefly, the object (a 30-cm long wooden spatula) was inserted into the cage through the mesh of the cage over the nest box for $30 \mathrm{~s}$ in each repetition. The observer assessed animal response to the object introduced into the living space. The procedures were carried out in triplicate (during the premating season - January/February) with a 2-week break between the consecutive repetitions of the test. The tests were performed early in the morning by one tester for all of the repetitions. The behavioural response of each individual was determined separately for each of the three replicates. The animal, which was classified at least twice as the same behaviour type in all three repetitions, was assigned to this profile as a final assessment. For example, if in subsequent repetitions the behavioural response was assessed as aggressive, curious and aggressive, the animal was eventually assigned to an aggressive behavioural profile group (Figure 1). None of the tested animals were classified to three different behavioural profiles. Animal response to the test was analysed as described below.

Four behavioural profiles among the tested animals: aggressive, curious, fearful and neutral were distinguished. Mink that were assessed as aggressive attacked the stick and grasped it with their teeth; there was (or not) hardbite, lockjaw and jerking (attempt to pull the object inside the cage). The animals were in almost continuous physical contact with the object (throughout the duration of the test). Mink defined as curious showed considerable interest in the object reflected in intense sniffing, gentle contact and grabbing, while no aggressive behaviours were observed. Mink considered as 


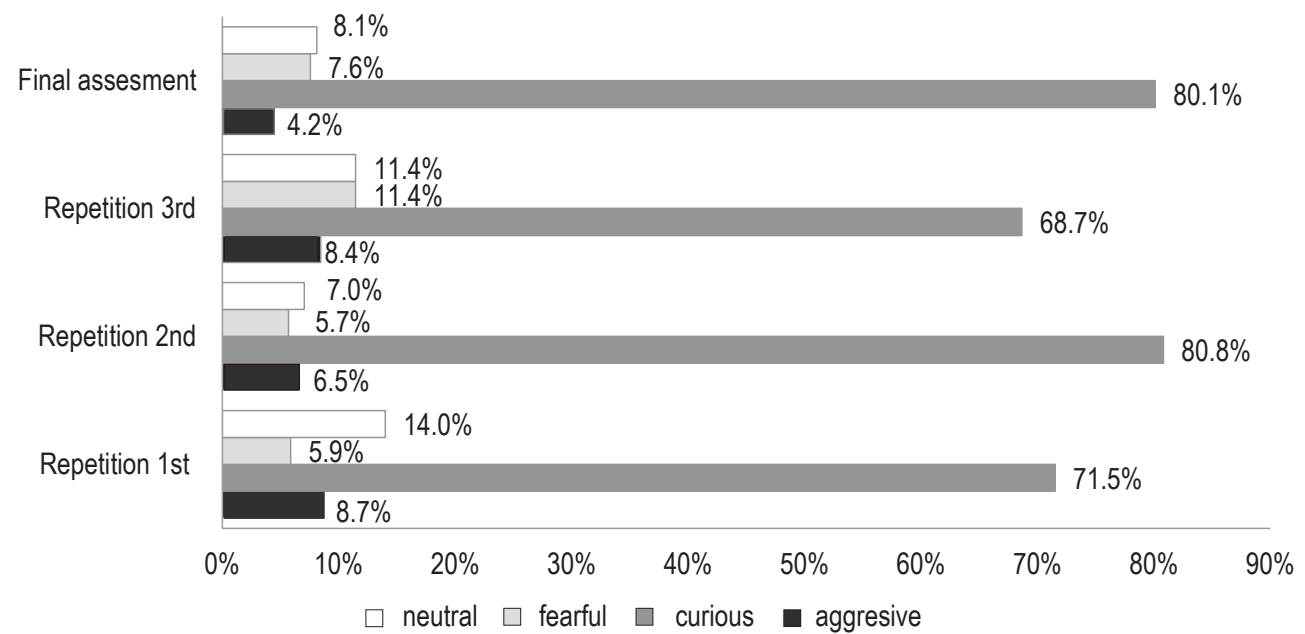

Figure 1. Percent distribution of animal response (neutral, fearful, curious, aggressive) in the stick test in three replicates compared with the final assessment of the behavioural profile; $n=576$ females of American mink (Neovision vision)

fearful showed little interest in the object (sniffing) and behaviours associated with fear prevailed: escape to the back of the cage, vocalization or defecation. Neutral mink were characterized by the lack of reaction to the introduced object. The animals observed the object during the test, but did not show any of the aforementioned types of behaviour.

One hundred one-year-old pastel females were selected from the tested group (see above) to study maternal behaviour. All of them were moved to another breeding house and kept individually to make the further observation easier. Due to the low frequency of neutral females as well as the high number of curious mink, all of the neutral ones and a corresponding number of females with other behaviour profiles were chosen for the test. The frequency of the behaviour profiles was as follows: $23 \%$ aggressive, $45 \%$ curious, $14 \%$ neutral and $18 \%$ fearful. The chosen mink were double-mated. The animals were observed during the perinatal period. The construction of the nest before the parturition and maternal behaviour (female care for the kits shortly after birth) were included in the analysis of female behaviour.

The preparation of the nest was assessed about two weeks before the expected parturition (approximately 10 to 17 days) taking into account the necessity of human intervention in its proper construction. The mink were provided with a proper amount of bedding material (oat straw and spruce scobs) placed inside the cage for assessment of the nesting behaviour. The features of the nest were assessed as follows: the amount of used straw, the way of construction (shape) as well as the visibility of the bottom of the nest box (metal mesh wire). The nest construction was categorized as (1) very good, with a large amount of straw used, a good shape, an invisible bottom, no need of human intervention; (2) medium, an moderate amount of straw used, a noticeable shape but not finished, slight human intervention required and (3) poor, a small, insufficient amount of straw, shapeless, a visible bottom of the nest, human intervention needed. In the case of the need for human intervention additional straw was added into the nest by the breeder.

Maternal behaviour was evaluated during the first week of life of young mink (day $4 / 5$ after delivery). The females were video recorded for 10 hours. Recordings were made with video cameras Sony HDR-CX240EB (Sony Europe B.V., Weybridge, UK). The cameras were placed on a tripod on the corridor of the pavilion and set to cover the whole segment to allow observation of the females (the tripod allowed the cameras to be set high and turned down straight to the cage and nestbox). No extra light was used. Due to the feeding period, the observations were divided into two equal periods: (1) before and (2) after feeding. The recordings were subjected to a very detailed analysis of the maternal behaviour of females. The following aspects were considered in the assessment of the mothers' care for the kits: the time spent by the females inside the nest as well as the number and time of carrying the young from the nest by the female. Each event of leaving the nest by the female and its duration was recorded. Then, the average duration of the absence from the nest in both observation periods was estimated.

The reproduction results of the examined animals were also taken into account in the assessment of maternal behaviour. The number of born mink was estimated at day 4 after birth and the number of reared mink was assessed at the age of 8 weeks. Data on the reproduction of the observed females 
(number of offspring born and raised) were collected from the farm's documentation.

During this stage of the experiment, none of the 100 mink were disqualified from the study (due to sickness, deaths, no delivery etc.). Moreover, the transferring of kits between the dams was not allowed in the experimental group.

The effect of mink behaviour during the behavioural tests on the results of reproduction was tested. The level of significance $(P<0.05)$ of the factors was verified by a multifactorial analysis of variance (GLM procedure) with the least squares method (LSM ) using the SAS statistical package version 9.4 (SAS Institute Inc., Cary, NC, USA).

The model included:

- the fixed effect of the emotional reactivity profile;

- the fixed effect of the age of the females at the time of the event;

- the fixed effect of the quality of the nest;

- regressions on the day of the recruitment counted from the beginning of the year.

The relationship between the behavioural profile and time spent by the females in the nest was assessed by a multivariate analysis of variance (GLM) with the LSM. The model took into account the fixed effect of the behavioural profile and the time spent by the females in the nest.

The relationship between the nest quality and the time spent by the females outside the nest was verified by a multivariate analysis of variance (GLM) with the LSM. The model took into account the fixed effect of the behavioural profile and the quality of the nest.

\section{Results}

The mink behaviour assessed by the stick test had a significant impact on the number of offspring born (present at day 4) and reared (8-week old). There was a significantly higher number of kits present at day 4 from the neutral females in comparison to aggressive ones (Table 1). The number of reared kits was significantly higher in the group of the fearful females than the number of offspring juveniles from the aggressive mink (Table 1).

The largest group among the assessed nests comprised medium quality nests $(60 \%)$ followed by very good quality nests (37\%). In turn, nests rated as poor quality and requiring intervention constituted $3 \%$. Due to their low number, the nests rated as poor quality were not included in the further analysis. There was no statistically significant difference in
Table 1. Number of kits present at day 4 and reared (8-week old) depending on the behavioural profile of the American mink (Neovision vision) female

\begin{tabular}{|c|c|c|c|c|}
\hline \multirow{3}{*}{$\begin{array}{l}\text { Behavioural } \\
\text { profile (n) }\end{array}$} & \multicolumn{4}{|c|}{ Number of kits } \\
\hline & \multicolumn{2}{|c|}{ present at day 4} & \multicolumn{2}{|c|}{ reared (8-week old) } \\
\hline & LSM & SE & LSM & SE \\
\hline Aggressive (23) & $4.70^{b}$ & 0.66 & $3.86^{b}$ & 0.63 \\
\hline Curious (45) & $5.65^{\mathrm{ab}}$ & 0.28 & $4.88^{\mathrm{ab}}$ & 0.27 \\
\hline Fearful (18) & $6.02^{\mathrm{ab}}$ & 0.69 & $5.87^{a}$ & 0.66 \\
\hline Neutral (14) & $6.89^{\mathrm{a}}$ & 0.64 & $5.37^{\mathrm{ab}}$ & 0.61 \\
\hline
\end{tabular}

LSM - least squares mean; SE - standard error; ${ }^{a, b}$ - values within each column marked with different letters are significantly different at $P \leq 0.05$

the number of born (present at day 4) and reared kits between females whose nests were rated as medium $(5.98 \pm 0.29$ and $5.11 \pm 0.28$, respectively for born and reared kits) and very good quality $(5.18 \pm 0.34$ and $4.38 \pm 0.32$, respectively). The very good quality nests were most often built by females assessed in the stick test as fearful; however there was no difference between fearful and neutral mink. The nests of the aggressive mink were most often referred to as medium quality in comparison to ones constructed by fearful females.

Table 2. Probability of nest construction with a certain quality depending on the behavioural profile of the American mink (Neovision vision)

\begin{tabular}{llllll}
\hline \multirow{2}{*}{$\begin{array}{l}\text { Behavioural } \\
\text { profile }(\mathrm{n})\end{array}$} & \multicolumn{2}{l}{ Nest quality } & & \\
\cline { 2 - 3 } \cline { 5 - 5 } & medium & & very good & \\
\cline { 2 - 3 } & LSM & SE & & LSM & SE \\
\hline Aggressive (23) & $0.75^{\mathrm{A}}$ & 0.14 & & $0.25^{\mathrm{B}}$ & 0.14 \\
Curious (45) & $0.54^{\mathrm{AB}}$ & 0.06 & & $0.38^{\mathrm{B}}$ & 0.06 \\
Fearful (18) & $0.25^{\mathrm{B}}$ & 0.14 & & $0.75^{\mathrm{A}}$ & 0.14 \\
Neutral (14) & $0.40^{\mathrm{AB}}$ & 0.13 & & $0.60^{\mathrm{AB}}$ & 0.13 \\
\hline
\end{tabular}

LSM - least squares mean; SE - standard error; ${ }^{A, B}$ - values within each column marked with different letters are significantly different at $P \leq 0.01$

After giving birth, females spent considerable amount of time taking care of their offspring, which involved feeding, warming and nursing. Given the foraging behaviour of mink, females have to split their time between nursing, protecting the nest and eating. According to the observation results it was noticed that the females stayed in the nest and left it regularly. Average time spent by the American mink females in the nest during the day in the context of the behavioural type of the female was analysed (Figure 2). Regardless of the behaviour profile the females spent similar amount of time with the young at the time before feeding (from 16.8 to $22.3 \mathrm{~min}$ ). After the feeding, the neutral females stayed with their offspring in the nest significantly longer (almost twice as long). 


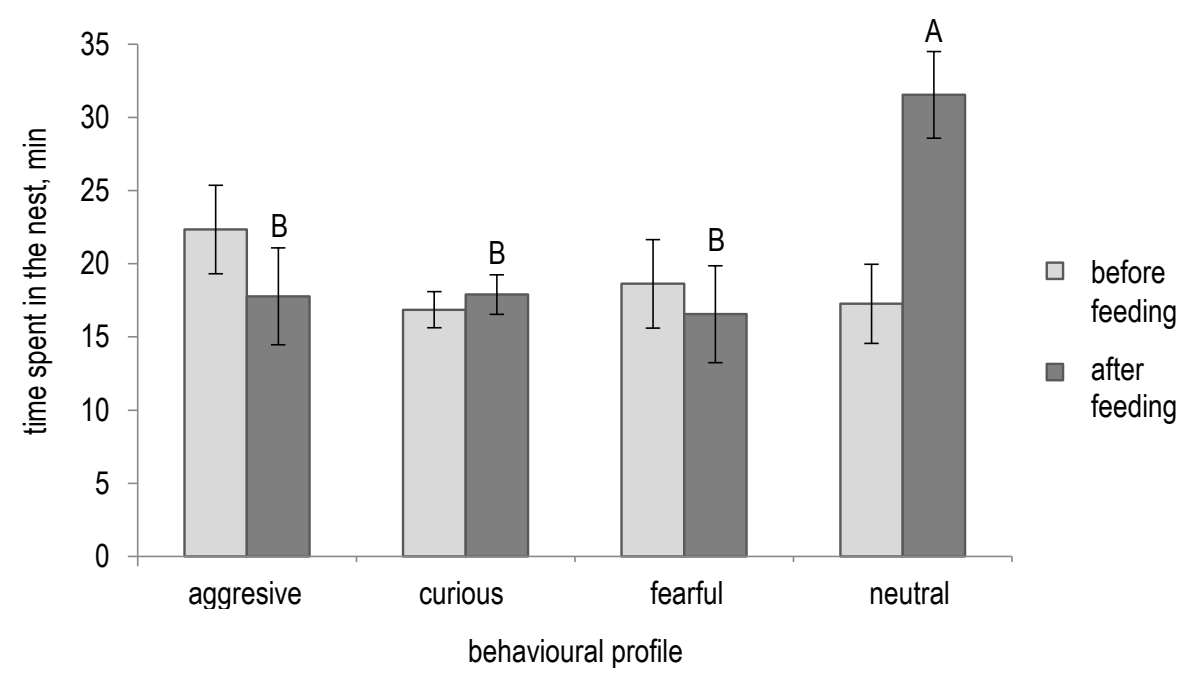

Figure 2. Average time spent by the American mink (Neovision vision) females in the nest depending on the behavioural profile (aggressive, curious, fearful and neutral) and period (before and after feeding)

The 10-hour observations were conducted at day 4 and 5 after delivery; ${ }^{A, B}$ - bars marked with different letters within each period are significantly different at $P \leq 0.01$

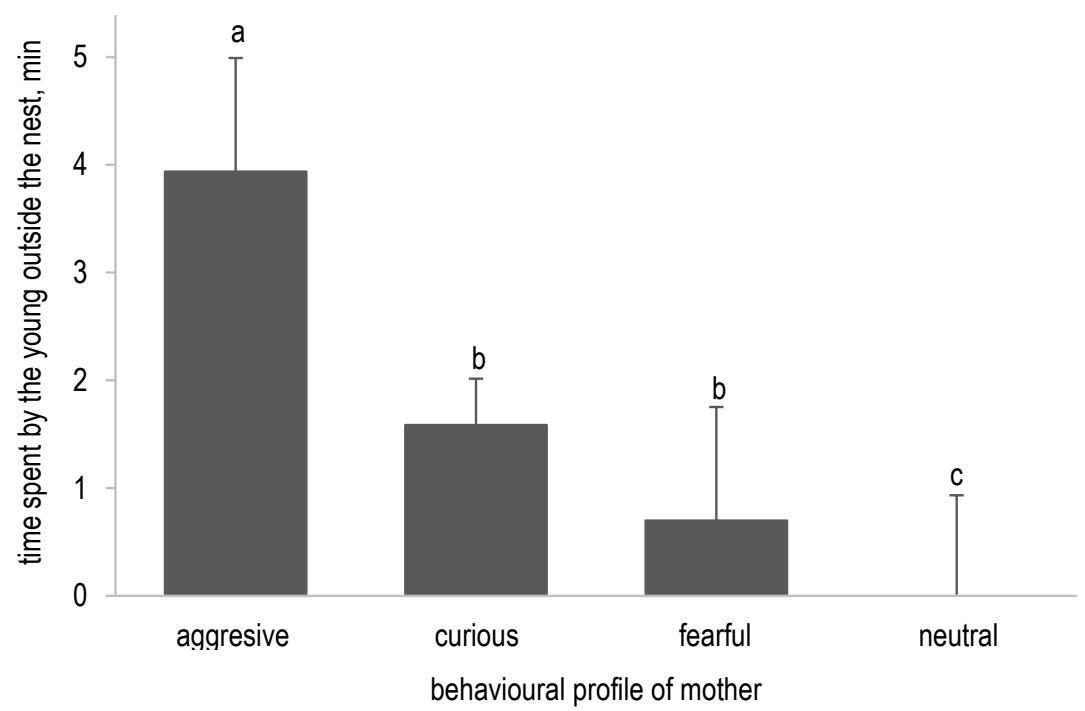

Figure 3. Average time ( $\mathrm{min}$ ) spent by offspring outside the nest depending on the mother's behavioural profile (aggressive, curious, fearful and neutral) during 10-hour observation at day 4 and 5 after delivery

a,b - bars marked with different letters are significantly different at $P \leq 0.05$

Before feeding, the longest time spent out of the nest was noted for females whose nests were assessed as weak and requiring human intervention $(P<0.01)$ in comparison to females whose nests were rated as medium and very good quality $(48.9,17.2$ and $15.9 \mathrm{~min}$, respectively). However, after the feeding period they spent significantly less time away from the young than the females whose nests were assessed as very good quality $(P<0.05)$.

During the observation, it was also noticed that some females carried the offspring outside the nest. The behavioural profile of the mother influenced the number and duration of those events. The aggressive females took the young out of the nest on average 12 times during the 10-hour observation. The curious females carried their offspring 6 times, the fearful mink - only 2 times, and only one neutral female took out the offspring (with one young, just in and out). The time spent outside the nest was significantly higher for the young born by the aggressive females in comparison to the offspring born by the females with other behavioural profiles (neutral, fearful and curious) $(P<0.05)$ (Figure 3$)$. Interestingly, the highest probability of taking the offspring outside the nest was obtained for females building the medium quality nest $(\mathrm{LSM}=2.51, \mathrm{SE}=0.49 ; P<0.01)$. 


\section{Discussion}

The results obtained during the investigations presented in this work indicate that females that were characterized as fearful in the stick test were characterised by the best breeding results - they reared on average almost 6 kits. The fearful females also obtained the best rates for the construction of the nest and the young were taken out only sporadically. The results obtained in this study are not consistent with the results presented by Łapiński et al. (2013), who showed that animal temperament did not affect the results of mink breeding. In addition, our results are also not in line with the research conducted by Korhonen et al. (2002), who found that the temperament dichotomy (confident vs fearful) in farmed mink stock had a marked effect on the reproductive performance of this species, i.e. a lower reproductive outcome was stated in fearful mink. Also, in the review of Wingfield and Sapolsky (2003) it was claimed that long-term chronic stress can led to a reduction in fertility in mammals. However, feeling anxiety by females may affect their behaviour in the postpartum period (fearful females). They are most effective in protecting themselves and their offspring from threats, which has a direct impact on their maternal behaviour, feeding the young, the time spent away from the young and ultimately on the number of raised offspring.

In contrast to the fearful females, those with an aggressive behavioural profile achieved unsatisfactory breeding results - an average of 3.86 kits were reared, which was the lowest result among the different behavioural profiles of animals selected in this study. This is directly related to the time the female spends with the young and the frequency of taking them out of the nest, which is determined by environmental factors during the rearing period (Amstislavsky and Ternovskaya, 2000). The frequent carrying of young mink outside the nest may result in lowering their immunity and, consequently, death, thus reducing the number of kits weaned from the female. However, Malmkvist et al. (2007) reported a high survival rate in a group of mink in which females took out the young from the nest on average 17 times for 3 min each time, while females with high mortality of offspring took them out on average 8 times for at least one minute.

The nest construction is crucial for the survival of young mink due to the specific microclimate created therein. Immediately after the birth of kits, the young cuddle each other (huddling effect) or in the mother's coat, which contributes to maintenance of proper body temperature (Malmkvist et al., 2007; Zschille et al., 2010). Young mink are unable to maintain the proper body temperature up to 22-29 days after birth (Harjunpää and RouvinenWatt, 2004). The huddling effect can last up to 14 days or even up to 30 days after birth depending on many factors, e.g., environmental conditions, litter size, access to feed (Tauson et al., 2006). Only after that time, kits open their eyes (Brandt et al., 2013) and start to separate themselves from the siblings in the same nest. The effectiveness of cuddling depends on the ambient temperature, the construction of the nest (the amount of used bedding) and the heat transfer from the female to the nesting offspring (Malmkvist et al., 2007). The temperature inside the nest, depending on the used material (straw, cut straw or mixed), varies from $20.8{ }^{\circ} \mathrm{C}$ to $26.6{ }^{\circ} \mathrm{C}$ (Malmkvist and Palme, 2008). Therefore, checking the construction of the nest before parturition and its possible correction by humans seems to be reasonable. The postnatal observations made in the present study showed that females with different behavioural profiles spent a similar amount of time inside the nest in the first period (before feeding), which may indicate that when the temperature is low in the morning the female warms her offspring with her body. Interestingly, the neutral females definitely spent the longest time outside the nest during the second period (after feeding) as compared to the other behavioural profiles. It should be noted here that the nests assessed in this study were inspected before the labour and, if necessary, supplemented by man. Despite the human intervention, the female mink were characterized by the considerable protectiveness toward their young. This indicates a strong natural instinct of females to prepare the nest properly by using natural insulation materials for nest construction and constant care of the young. Malmkvist and Palme (2008) showed that access to straw before birth had a positive impact on the delivery, while the use of a plastic insert, which is widely used on farms, did not give such an effect. Hansen et al. (2010) suggested that the survival rate of kits during the suckling period may be improved by selection for the maternal effect on kit survival. The better scores for the nest construction by the fearful mink may suggest that they have a stronger need to isolate themselves and their juveniles from any form of stress stimuli (human, predators) in comparison to other behavioural profiles. 


\section{Conclusions}

Knowledge of the behavioural profile of female mink seems to be of a great importance, as significant differences in the number of offspring born and reared depending on the behavioural profile of mothers exist. The construction of the nest before delivery can also be an important factor differentiating the female mink. Despite the tendency to eliminate fearful behaviours from farms, it should be noted that the animals with this behavioural profile have significantly higher breeding results than aggressive ones. However, further research explaining differences in results from previous studies is needed.

\section{References}

Amstislavsky S., Ternovskaya Y., 2000. Reproduction in mustelids. Anim. Reprod. Sci. 60-61, 571-581, https://doi.org/10.1016/ S0378-4320(00)00126-3

Axelsson H.M., Hansen S.W., Loberg J., Lidfors L., 2017. Effects of group size on behaviour, growth and occurrence of bite marks in farmed mink. Appl. Anim. Behav. Sci. 194, 112-119, https:// doi.org/10.1016/j.applanim.2017.05.008

Brandt C., Malmkvist J., Nielsen R.L., Brande-Lavridsen N., Surlykke A., 2013. Development of vocalization and hearing in American mink (Neovison vison). J. Exp. Biol. 216, 3542-3550, https://doi.org/10.1242/jeb.080226

Dawson L., Buob M., Haley D., Miller S., Stryker J., Quinton M., Mason G., 2013. Providing elevated 'getaway bunks' to nursing mink dams improves their health and welfare. Appl. Anim. Behav. Sci. 147, 224-234, https://doi.org/10.1016/j. applanim.2013.04.001

Díez-León M., Quinton M., Mason G., 2017. How tall should a mink cage be? Using animals' preferences for different ceiling heights to improve cage design. Appl. Anim. Behav. Sci. 192, 24-34, https://doi.org/10.1016/j.applanim.2017.03.002

Felska-Błaszczyk L., Lasota B., Seremak B., 2016. Conception rates in farm mink (Neovison vison) in relation to first mating date, age and color variety. Anim. Sci. J. 87, 873-877, https://doi. org/10.1111/asj.12517

Gugołek A. (Editor), 2011. Nutritional Recommendations and Nutritional Value of Feed. Fur Animals (In Polish). The Kielanowski Institute of Animal Physiology and Nutrition PAS. Jabłonna (Poland)

Hansen B.K., Su G., Berg P., 2010. Genetic variation in litter size and kit survival of mink (Neovison vison). J. Anim. Breed. Genet. 127, 442-451, https://doi.org/10.1111/j.1439-0388.2010.00872.x

Harjunpää S., Rouvinen-Watt K., 2004. The development of homeothermy in mink (Mustela vison). Comp. Biochem. Physiol. A. Mol. Integr. Physiol. 137, 339-348, https://doi. org/10.1016/j.cbpb.2003.10.015

Korhonen H.T., Jauhiainen L., Rekilä T., 2002. Effect of temperament and behavioural reactions to the presence of a human during the pre-mating period on reproductive performance in farmed mink (Mustela vison). Can. J. Anim. Sci. 82, 275-282, https:// doi.org/10.4141/A01-088
Łapiński S., Bzymek J., Niedbała P., Migdał Ł., Zoń A., Lis M., 2013. Effect of age and temperament type on reproductive parameters of female raccoon dogs (Nyctereutes procyonoides Gray). Ann. Anim. Sci. 13, 807-814, https://doi. org/10.2478/aoas-2013-0021

Malmkvist J., Gade M., Damm B.I., 2007. Parturient behaviour in farmed mink (Mustela vison) in relation to early kit mortality. App. Anim. Behav. Sci. 107, 120-132, https://doi. org/10.1016/j.applanim.2006.09.018

Malmkvist J., Hansen S.W., 2002. Generalization of fear in farm mink, Mustela vison, genetically selected for behaviour towards humans. Anim. Behav. 64, 487-501, https://doi.org/10.1006/ anbe.2002.3058

Malmkvist J., Palme R., 2008. Periparturient nest building: Implications for parturition, kit survival, maternal stress and behaviour in farmed mink (Mustela vison). App. Anim. Behav. Sci. 114, 270-283, https://doi.org/10.1016/j.applanim.2008.01.018

Malmkvist J., Sørensen D.D., Larsen T., Palme R., Hansen S.W., 2016. Weaning and separation stress: maternal motivation decreases with litter age and litter size in farmed mink. App. Anim. Behav. Sci. 181, 152-159, https://doi.org/10.1016/j. applanim.2016.05.028

Mandalaywala T.M., Higham J.P., Heistermann M., Parker K.J., Maestripieri D., 2014. Physiological and behavioural responses to weaning conflict in free-ranging primate infants. Anim. Behav. 97, 241-247, https://doi.org/10.1016/j. anbehav.2014.09.016

Mason G.J., Latham N.R., 2004. Can't stop, won't stop: is stereotypy a reliable animal welfare indicator? Anim. Welf. 13, Suppl. 1, S57-S69

Meagher R., Bechard A., Palme R., Díez-León M., Hunter B.D., Mason G., 2012. Decreased litter size in inactive female mink (Neovison vison): Mediating variables and implications for overall productivity. Can. J. Anim. Sci. 92, 131-141, https:// doi.org/10.4141/cjas2011-107

Newberry R.C., Swanson J.C., 2008. Implications of breaking motheryoung social bonds. App. Anim. Behav. Sci. 110, 3-23, https:// doi.org/10.1016/j.applanim.2007.03.021

Ślaska B., Rozempolska-Rucińska I., 2011. Mating system and level of reproductive performance in mink (Neovison vison). Ann. Anim. Sci. 11, 105-113

Ślaska B., Rozempolska-Rucińska I., Jeżewska-Witkowska G., 2009. Variation in some reproductive traits of mink (Neovison vison) according to their coat colour. Ann. Anim. Sci. 9, 287-297

Tauson A.-H., Chwalibog A., Tygesen M.P., 2006. Late development of homoeothermy in mink (Mustela vison) kits - a strategy for maximum survival rate. J. Anim. Physiol. Anim. Nutr. 90, 38-45, https://doi.org/10.1111/j.1439-0396.2005.00617.x

Wingfield J.C, Sapolsky R.M., 2003. Reproduction and resistance to stress: When and how. J. Neuroendocrinol. 15, 711-724, https://doi.org/10.1046/j.1365-2826.2003.01033.x

Zschille J., Stier N., Roth M., 2010. Gender differences in activity patterns of American mink Neovison vison in Germany. Eur. J. Wildl. Res. 56, 187-194, https://doi.org/10.1007/s10344-0090303-2 